\title{
Reward and punishment effects on error processing and conflict control
}

\author{
Birgit Stürmer ${ }^{1}{ }^{*}$, Roland Nigbur ${ }^{1}$, Annekathrin Schacht ${ }^{2}$ and Werner Sommer ${ }^{1}$ \\ ' Department of Psychology, Humboldt-Universität zu Berlin, Berlin, Germany \\ ${ }^{2}$ Courant Research Centre Text Structures, University of Göttingen, Göttingen, Germany
}

Edited by:

Tom Verguts, Ghent University,

Belgium

Reviewed by:

Thomas Kleinsorge, Leibniz Research Centre for Working Environment and

Human Factors, Germany

Jelle Demanet, Ghent University,

Belgium

Henk Van Steenbergen, Leiden

University, Netherlands

*Correspondence:

Birgit Stürmer, Institute for

Psychology, Humboldt-Universität zu

Berlin, Rudower Chaussee 18, 12489

Berlin, Germany.

e-mail: birgit.stuermer@cms.

hu-berlin.de
Recently, positive affect has been reported to reduce cognitive conflicts and adaptations related to conflict control. van Steenbergen et al. (2009) proposed that the aversive quality of conflicts drives short-term adaptations following a conflict. They reasoned that monetary gain and its positive emotional consequences might counteract the aversive quality of conflict and hence reduce subsequent adaptations. In two experiments, we combined Simon-type conflicts with monetary gains and losses in between trials and analyzed eventrelated brain potentials. In Experiment 1, gains and losses occurred randomly between trials as a lottery, whereas in Experiment 2 gains and losses were contingent upon performance, either rewarding the $25 \%$ fastest responses or penalizing the $25 \%$ slowest responses. In Experiment 1, conflict adaptation was completely unaffected by gains or losses; contrary to predictions, in Experiment 2, conflict adaptation in reward blocks was more pronounced after a gain. In Experiment 2 we also investigated the error-related negativity (ERN) - a brain signal proposed to be related to performance monitoring. The ERN and behavioral post-error slowing were enlarged in the context of reward; therefore, reward increases error adaptation, possibly by enhancing the subjective value of errors. In conclusion, affective modulations of conflict adaptations seem to be much more limited than previously asserted and adaptive mechanisms triggered by errors and conflicts dissociate.

Keywords: conflict adaptation, error processing, reinforcement, reward, punishment, event-related brain potentials, error-related negativity

\section{INTRODUCTION}

Despite many years of research on cognitive conflicts, the potential role of emotions or affects in these situations is taken into account only recently. The present study investigates the effect of rewardinduced motivational states on one element of cognitive control during conflict processing - action monitoring. Monitoring one's own actions is a critical precondition for adaptive behavior in general and for handling cognitive conflicts in particular.

The original conflict monitoring account (Botvinick et al., 2001) postulated that the dorsal anterior cingulate cortex (ACC) responds to conflicts, arising during various tasks, by issuing a conflict signal. This conflict signal triggers strategic adjustments in cognitive control by redirecting attention according to the task demands. Thus, the ACC would serve as a conflict monitoring device. The conflict monitoring theory stimulated research interests in sequential dependencies during conflict processing. In typical interference tasks like the Stroop, Flanker, or Simon task, a set of multidimensional stimuli is assigned to a set of responses. Usually, only one stimulus dimension is task-relevant, while other stimulus dimensions are task-irrelevant but at least one of them shares features with the relevant dimension (Lu and Proctor, 1995). In the Simon task, for example, left or right-hand responses are performed as a function of a non-spatial stimulus feature (S; e.g., shape or color) while the stimuli are presented either on the left or right-side. Responses (R) are faster and more accurate when $\mathrm{S}$ and $\mathrm{R}$ locations correspond (C, compatible events) than when they do not correspond (IC, incompatible events). In general, incompatible trials provoke conflicts in information processing when at least one feature of $\mathrm{S}$ or $\mathrm{R}$ contradicts the correct response. For example, in the Simon task, the stimulus may activate a right-side response by virtue of its (irrelevant) location, which contradicts a left-side response demanded by the relevant stimulus dimension, resulting in slower and more error-prone responses.

In most kinds of tasks with S-R conflicts, conflict-strength in the current trials depends on the correspondence condition of the preceding trials: after non-corresponding events conflicts are much smaller than after corresponding events (Gratton et al., 1992; Stürmer et al., 2002; Egner et al., 2007). According to the conflict monitoring approach, conflicts redirect the attentional focus to task-relevant features, diminishing the influence of task-irrelevant features, hence reducing the conflicts between these features and the response on the next trial.

In a recent extension of his conflict monitoring account, Botvinick (2007) suggested that the processing of conflicts is effortful and therefore aversive. Hence, conflicts should bias decision-making toward more efficient task strategies. The presumable redirection of attention after a conflict trial may be a direct consequence of the aversive efforts in dealing with this conflict. If conflict adaptation behavior is triggered by the negative affect elicited by conflicts, one may presume that positive affect would counteract conflict adaptation. This assumption is supported by findings of Kuhl and Kazen (1999), showing that the 
Stroop effect is largely reduced by the short-term induction of positive affect. Following the suggestion that conflicts are experienced as negative, van Steenbergen et al. (2009) reasoned that monetary gain and its positive emotional consequences might counteract the aversive quality of the preceding conflict and hence reduce subsequent conflict-driven adaptation processes. Indeed, in a flanker task a small but significant reduction of conflict adaptation was found after monetary gain. In a follow-up study, van Steenbergen et al. (2010) applied mood induction and showed that as a trendless positive mood induction tended to be associated with larger conflict-related adaptation. Taken together, the authors concluded that affect adaptively regulates cognitive control.

A direct link between affective and cognitive processing in conflict control was already implied in the seminal model by Miller and Cohen (2001). In this account, the prefrontal cortex (PFC) establishes S-R mappings by biasing competition between conflicting sensory inputs or motor outputs to favor relevant aspects for current task-performance. Phasic dopamine (DA) release by the midbrain DA system plays a major role in gating the appropriate update of task-relevant goal representations in the PFC. Thus, reward-driven DA release related to the reward prediction error (Schultz, 1998) is proposed to strengthen top-down control over bottom-up processing. One could therefore assume that conflict adaptation as a top-down control process should be enhanced after a reward when DA is released.

This assumption is in line with studies investigating conflict adaptation in Parkinson's disease (PD) patients who suffer from a low level of midbrain DA. Conflict adaptation in a Simon interference task was much reduced in PD patients (Praamstra and Plat, 2001; Fielding et al., 2005). These findings contrast with the view of van Steenbergen et al. (2009) who claimed that DA bursts were responsible for the reduced conflict adaptation observed in the context of reward.

A further problematic point for the idea that conflict adaptation is triggered by the aversiveness of the efforts involved in cognitive conflict processing is the lack of evidence for the purported negative emotional valence of cognitive conflicts. As shown by Schacht et al. (2010) in a direct comparison of Go/Nogo and Simon tasks, the emotions elicited by conflicts are task specific and not necessarily aversive. Emotional responses, indicated in a number of psychophysiological parameters, were only present in Go/Nogo conflicts but not in incompatible Simon task trials. Moreover, the construal of conflicts as aversive, adaptation-driving events was further called into question by indications that the emotions elicited in Nogo conflicts seem to be appetitive rather than aversive (cf. Schacht et al., 2009).

In sum, the theoretical predictions for the relationship between affect and conflict processing are controversial. Whereas van Steenbergen et al. (2009) hold that conflict adaptation is triggered by the aversive nature of conflict processing, other findings indicate that conflict adaptation should be facilitated by emotionally positive, DA-releasing events or states.

Here we tested in two experiments with the Simon task whether reward counteracts the presumably negative experience of a conflict and hence reduces conflict adaptation as predicted by van Steenbergen et al. (2009). In Experiment 1, reward was presented non-contingent to behavior, attempting to replicate the study of van Steenbergen et al. (2009) with a different conflict task. Because the predicted effect was absent, Experiment 2 explored the effects of presenting reward and punishment contingent upon performance.

\section{EXPERIMENT 1}

In the first experiment, a Simon task was combined with wins or losses in between two Simon trials. Wins and losses were not related to participant's performance but were presented at random, closely replicating the flanker task study van Steenbergen et al. (2009). In the present experiment, one of two stimuli appeared above or below fixation and required a choice response according to the stimulus shape on an upper or lower key. This procedure was similar to that of Stürmer et al. (2002) where context-driven adaptation had been present also when direct trial repetitions were excluded. Usually, responses to direct trial repetitions are very fast and confound with sequential effects related to conflict adaptation. In order to avoid these simple priming mechanisms unrelated to cognitive control (Mayr et al., 2003), we excluded direct repetitions by experimental design.

To control whether the affective manipulation by gain signals was effective, we recorded event-related brain potentials (ERPs) to the win and loss signals presented in between Simon trials (Holroyd et al., 2008). In contrast to van Steenbergen et al.'s (2009) study where gain signals directly followed the responses, we inserted a 500-ms interval after responses to avoid an overlap of response-related and gain signal-related ERPs. To guarantee a suitable baseline for ERP analyses we extended the inter-trial interval from 200 to $400 \mathrm{~ms}$, as used by van Steenbergen and colleagues, to $1000 \mathrm{~ms}$ in the present study. Moreover, to ensure a positive payoff at the end of the experiment - maintaining participant's motivation - monetary gains per trials exceeded the losses by $0.05 €$. Slightly higher wins than losses had the additional advantage that an influence of rewards on conflict adaptation was emphasized.

\section{METHOD AND MATERIAL \\ Participants}

Twenty-one neurotypical adults (age range $=20-49$ years, $M=28.5$ years, $\mathrm{SD}=9.2$ years; 4 males) participated in the experiment. All were right-handed (handedness score $=75.6$ ) with normal or corrected-to-normal vision. Data of one further participant had to be discarded due to excessive error rates ( $>30 \%)$. Prior to testing, participants provided written consent according to the declaration of Helsinki and completed a handedness questionnaire (Oldfield, 1971).

\section{Experimental setup and design}

Participants were seated in a sound-attenuated and dim lit electrically shielded chamber. All stimuli were presented on a $17^{\prime \prime}$ monitor of a Pentium processor using Presentation software, at a viewing distance of approximately $80 \mathrm{~cm}$.

Participants responded with left and right index fingers to the shape of the stimuli using two vertically aligned response keys. In compatible trials stimulus and response position corresponded whereas in incompatible trials they did not. The stimuli consisted of a white square and rhombus, presented randomly above or below a central fixation point against a gray background. 
Each trial started with the presentation of a central fixation point for $1000 \mathrm{~ms}$, followed by the Simon stimulus shown for $200 \mathrm{~ms}$ approximately $1.5 \mathrm{~cm}$ above or below the fixation point. After a practice block participants were informed that they could earn between 10 and $20 €$ via a lottery algorithm that would provide gains or losses independently of their performance. The gain signals appeared $500 \mathrm{~ms}$ after the response (or $1.5 \mathrm{~s}$ after the stimulus in case of an omission) and consisted of a green, red, or blue circle (all $1.2 \mathrm{~cm}$ in diameter) displaying a monetary win $(+0.25 €)$, a loss $(-0.20 €)$, or a blank $(0.00 €)$, respectively. Gain signals (win, loss, or blank) were presented centrally for $500 \mathrm{~ms}$. Win, loss, and blank feedbacks appeared randomly with equal probabilities. After 60 practice trials, eight blocks of 120 trials were presented.

\section{EEG recording and processing}

The EEG data was recorded from 60 electrodes placed in an electrode cap and referenced to the left mastoid with a bandpass of $0.01-250 \mathrm{~Hz}$ at a sampling rate of $500 \mathrm{~Hz}$. Vertical and horizontal electro-oculograms (EOG) were recorded from external electrodes. Electrode impedance was kept below $5 \mathrm{k} \Omega$, using ECI electrode gel. Offline, continuous data were down-sampled to $250 \mathrm{~Hz}$, re-referenced to an average mastoid reference; blink correction was applied using independent component analyses as implemented in Brain Vision Analyzer. Artifacts were automatically rejected, eliminating epochs with voltage steps per sampling point $>50 \mu \mathrm{V}$, and low activity $(<0.5 \mu \mathrm{V})$ within a $100-\mathrm{ms}$ window. Offline, data were filtered, using Butterworth Zero Phase Filters (Time Constant: $3.18 \mathrm{~s}, 48 \mathrm{~dB} /$ oct; High Cut-off: $30 \mathrm{~Hz}$, $48 \mathrm{~dB} /$ oct). ERPs related to the gain signals were segmented into $1200 \mathrm{~ms}$ epochs, starting $200 \mathrm{~ms}$ before stimulus presentation; a 100-ms pre-stimulus baseline was applied.

\section{RESULTS}

\section{Performance}

Errors occurred in $4.1 \%$ of all trials (compatible $=2.4 \%$; incompatible $=5.7 \%$ ). An ANOVA on error rate including the factors compatibility (2 - C, IC) and gain signal (3 - win, blank, or loss) yielded a significant main effect of compatibility; $F(1,20)=32.72$, $p<0.001$. Neither the main effect of gain signal, nor the interaction of feedback and compatibility were significant, $F_{s}<1$.

In order to test the influence of different gain signals on conflict adaptation, we analyzed correct trials following correct trials and separated them according to their current compatibility, the compatibility of the preceding trial, and the feedback following the preceding trial. Only RTs $>100 \mathrm{~ms}$ entered into subsequent analyses. An ANOVA on RTs with factors compatibility (2 - C, IC), predecessor (2 - C, IC), and gain signal ( 3 - win, blank, or loss) yielded a significant main effect of compatibility, $F(1,20)=47.69$, $p<0.001$, a main effect of predecessor, $F(1,20)=25.18, p<0.001$, and a significant interaction of compatibility and predecessor, $F(1,20)=117.64, p<0.001$. However, there was neither a main effect of feedback nor an interaction with any of the other factors (see Table 1), Fs $<1$. Following van Steenbergen et al. (2009), we calculated a conflict adaptation measure that integrates the speed-up and slow-down of compatible and incompatible trials using the formula $[(\mathrm{CI}-\mathrm{CC})-(\mathrm{II}-\mathrm{IC})]$. Mean conflict adaptation scores (see Table 1) for trials after loss, win, and blank signals were 73,70 , and $81 \mathrm{~ms}$, respectively. An ANOVA on the
Table 1 | Reaction times (RT) and error rates (ER) as a function of condition in Experiment 1.

\begin{tabular}{lcc}
\hline Trial type & RT in ms (SD) & ER (SD) \\
\hline WIN SIGNAL & & \\
Compatible preceding compatible (cC) & $420(74.9)$ & $0.7(1.8)$ \\
Incompatible preceding compatible (iC) & $470(81.9)$ & $3.7(3.2)$ \\
Compatible preceding incompatible (cl) & $487(80.7)$ & $7.2(5.4)$ \\
Incompatible preceding incompatible (il) & $466(85.7)$ & $3.9(3.5)$ \\
Simon effect & $32(21.5)$ & $3.4(3.0)$ \\
Conflict adaptation effect & $70(29.0)$ & $6.2(5.9)$ \\
BLANK SIGNAL & & \\
Compatible preceding compatible (cC) & $417(75.5)$ & $1.5(2.2)$ \\
Incompatible preceding compatible (iC) & $471(87.8)$ & $3.8(4.5)$ \\
Compatible preceding incompatible (cl) & $494(84.3)$ & $7.9(6.1)$ \\
Incompatible preceding incompatible (il) & $467(88.0)$ & $3.7(3.7)$ \\
Simon effect & $37(25.0)$ & $3.2(2.7)$ \\
Conflict adaptation effect & $81(37.9)$ & $6.5(5.8)$ \\
LOSS SIGNAL & & \\
Compatible preceding compatible (cC) & $420(76.6)$ & $1.0(1.7)$ \\
Incompatible preceding compatible (iC) & $472(80.6)$ & $3.8(4.3)$ \\
Compatible preceding incompatible (cl) & $487(82.4)$ & $7.6(6.6)$ \\
Incompatible preceding incompatible (il) & $466(88.7)$ & $3.5(3.3)$ \\
Simon effect & $30(21.1)$ & $3.1(3.1)$ \\
Conflict adaptation effect & $73(44.8)$ & $6.9(7.7)$ \\
& &
\end{tabular}

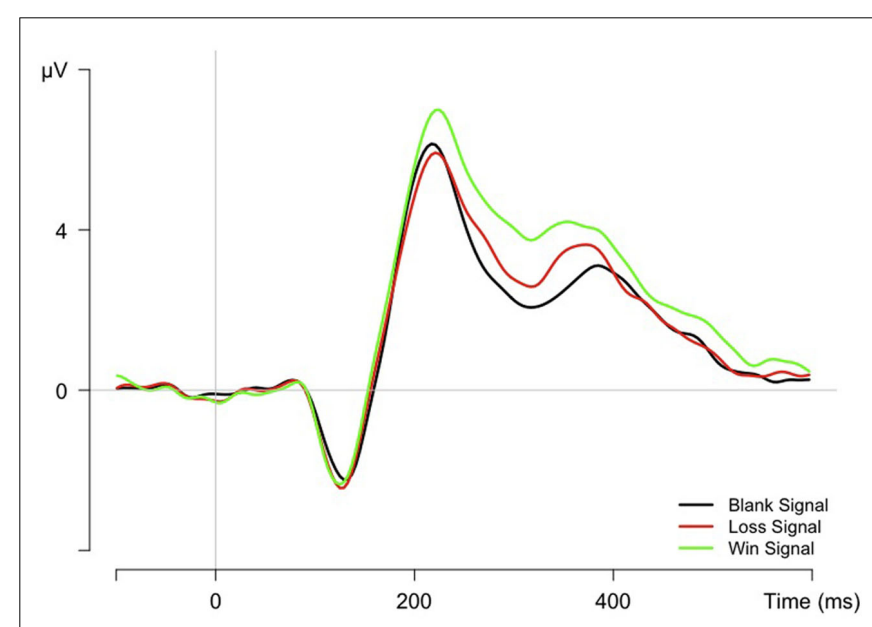

FIGURE 1 | Grand average ERPs at FCz electrode time-locked to gain signal presentation after correct trials.

conflict adaptation measure with the factor preceding gain signal (3 - win, blank, or loss) did not yield any significant differences, $F(2,40)=1$.

\section{Event-related potentials}

Feedback-related ERPs were analyzed on mean amplitudes at the FCz electrode between 225 and 275 ms after gain signal onset. In this time interval win signals elicited a significantly larger positivity $(2.3 \mu \mathrm{V})$ as compared to blank $(1.8 \mu \mathrm{V})$ or loss signals $(1.7 \mu \mathrm{V})$, $F(2,22)=7.3, p<0.01$ (see Figure 1). 


\section{DISCUSSION}

In the present Simon task, we found a reliable conflict adaptation effect of $75 \mathrm{~ms}$ in RTs. Although the conflict adaptation effect in the present study was far bigger than in the studies by van Steenbergen et al. (2009, 2010), in direct contradiction to their findings, it was unaffected by the type of preceding gain signal.

Our feedback manipulation was efficient as demonstrated by differential effects on the gain signal-related ERPs. Wins elicited more positive-going ERP deflections following feedback onset as compared to losses or blank feedback. This finding is in line with the feedback-correct related positivity as reported for example by Holroyd et al. (2008). Obviously, at least win signals were registered by the cognitive system of our participants. Therefore, the absence of affective modulation of conflict adaptation in the present experiment cannot be ascribed to an ineffective affective manipulation.

Possible reasons as to the discrepancy with the findings of van Steenbergen et al. $(2009,2010)$ will be elaborated in the General Discussion. In brief, one reason why motivationally significant stimuli were ineffective to modulate conflict adaptation here might be due to their unrelatedness to performance. It is conceivable that affect modulates conflict processing only if a direct connection between prior performance and reward/punishment can be made. Therefore, we conducted a second experiment where monetary gain and loss was contingent upon performance.

\section{EXPERIMENT 2}

In the two halves of this experiment, we either rewarded fast and correct responses or penalized slow responses. In case the proposed relationship between affect and conflict processing holds we assumed that affective modulations of behavioral adaptation effects would be present if reward and punishment were contingent upon performance. In addition, the specific influence of feedback-induced affect on reinforcement monitoring and error processing was investigated by analyzing the feedbackrelated negativity (FRN) and the error-related negativity (ERN) in the ERP.

The FRN in response to the reinforcement signal should indicate whether the performance-contingent feedback was registered by the participants. The FRN was first demonstrated in response to external feedback about incorrect responses (Miltner et al., 1997). It is elicited approximately $250 \mathrm{~ms}$ after the feedback stimulus with a fronto-medial scalp distribution. However the FRN was also present for feedback stimuli about losses and was larger than to gain signals (see Simons, 2010, for an overview).

Additionally, Experiment 2 investigated the influence of motivational states on cognitive control in error processing, which was possible here because error rates were larger than in Experiment 1 due to higher response speeds. Errors usually result in slower responses in the following correct trial. This so-called posterror slowing (PES) is seen as a compensatory control mechanism improving performance in subsequent trials (Gehring and Fencsik, 2001). According to the conflict monitoring account (Botvinick et al., 2001) errors result in strategic adaptations by increasing the response threshold in the next trial in order to reduce the likelihood of an upcoming error. Errors and its consequences are, hence, seen to affect cognitive control processes related to performance monitoring, similar to response conflicts in interference tasks (Yeung et al., 2004; but see Masaki et al., 2007; Notebaert et al., 2009, for an alternative view).

A prominent ERP component related to error processing is the ERN ( $\mathrm{Ne}$ or ERN). The ERN is tightly linked to the incorrect response, starts with the erroneous response and peaks about $60 \mathrm{~ms}$ later (Leuthold and Sommer, 1999; Falkenstein et al., 2000). The main generator of the ERN is probably located within the ACC (Ridderinkhof et al., 2004) and closely related to the midbrain DA system. Holroyd and Coles (2002) argue that, like negative feedback, response errors induce a dip in DA-cell firing (Schultz, 1998) which transmits to the ACC. The resulting disinhibition of ACC neurons is proposed to generate the ERN. Usually, larger ERN amplitudes are associated with improvements in performance monitoring (Larson et al., 2007, 2009; Olvet and Hajcak, 2008). Findings of Larson and Clayson (2011) suggest that increases in ERN amplitude are related to more focused attention that improves executive functions.

The involvement of the midbrain DA system in the processing of motivationally salient events implicates a relation between ERN and affective processing as confirmed by numerous reports. Larson et al. (2006) showed that the ERN is larger to errors within a background of pleasant emotional pictures as compared to neutral or unpleasant pictures. They suggest that the positive affective context creates a mismatch to task errors, thus enlarging the ERN. The ERN amplitude might reflect the subjective value of an error as derived from the recent reward history (Holroyd and Coles, 2008). Ogawa et al. (2011) used feedback signals of personal relevance (the trainer's voice in members of a university tennis team) and found that verbal admonishments significantly reduced ERN amplitude. However, there are also reports of enlarged ERNs in the context of negative affect. Wiswede and colleagues induced emotions either by presenting IAPS pictures (Wiswede et al., 2009a) or by embodied emotions, that is, participants had to hold a chop stick with their lips making them either smile or not smile (Wiswede et al., 2009b). The ERN was reduced in the no-smile conditions and in a context of negative IAPS pictures. However, embodied emotions did not show any effects on behavioral performance, casting doubt on whether performance monitoring was affected.

To sum up, when affective manipulations showed an influence on behavioral performance in conflict and error processing (e.g., Larson et al., 2006), the ERN was enlarged in a positive affective context. In the present experiment, feedback was provided according to participants' performance, which should strengthen the link between affective and cognitive processing. We therefore expected an affective modulation of conflict control and error processing. The ERN as an indicator of performance monitoring in errors should be enlarged in the context of rewards. Moreover, PES as a consequence of performance monitoring after preceding errors should increase as well. Predictions for the modulation of conflict adaptation by affective states are not univocal. Assuming that the aversiveness of a conflict triggers conflict adaptation (van Steenbergen et al., 2009), we should observe reduced conflict adaptation in the context of reward when the positive experience counteracts the conflict experience. 


\section{METHOD AND MATERIAL Participants}

Twenty-six neurotypical participants took part in the experiment; four of them were excluded (one had received wrong instructions and the others produced error rates $>20 \%$ in one of the experimental conditions). Of the remaining 22 participants (mean age $=24.6$ years), 12 were female and all were right-handed (mean handedness score $=92.2$; Oldfield, 1971). All participants reported normal or corrected-to-normal vision. Participants were informed in advance that they would receive at least $25 €$ for the 3.5 -h session and all gave their informed consent to the study.

\section{Experimental setup and design}

The experimental setup was identical to Experiment 1 with the following exceptions. The participants' head rested on a forehead and chin rest at a distance of $80 \mathrm{~cm}$ to a computer screen within an eye-tracking device, providing a constant viewing distance. Participants always started with a practice block of 240 Simon trials without any feedback. Afterward either the punishment or reward block followed; each contained 720 trials with self-paced breaks after every 60 trials. The timing of stimuli within a trial was identical to Experiment 1.

The order of punishment and reward blocks, the mapping of hands to response keys (index fingers of right and left hand on the upper or lower response keys) as well as the stimulus-response mapping in the Simon task (pressing the upper or lower key in response to the square or diamond) were counter-balanced across participants. For a given participant, the hands-to-key assignment and the stimulus-response mapping were constant throughout the experiment. Stimuli were presented in randomized order and direct repetitions of both stimulus form and stimulus location were excluded by design. All four possible sequences of compatible and incompatible trials and compatibility of their predecessors were equiprobable.

A staircase algorithm controlled that the $25 \%$ slowest responses were penalized in the punishment block and the $25 \%$ fastest responses received a bonus in the reward block. Four separate algorithms were calculated for compatible and incompatible trials and each response hand, respectively. To this end, reaction times were monitored online over the last 40 trials by a staircase algorithm starting at the $25 \%$-values of the practice block. This allowed calculating immediately after each response whether RT was among the $25 \%$ slowest or among the $25 \%$ fastest responses, respectively. An additional algorithm controlled how often the participant had received a gain or a loss on the 8 most recent trials. Whenever gain or loss signals were presented more or less often than in $25 \%$ of these 8 trials (i.e., 2 trials out of 8 ), the gain or loss rate was temporarily adjusted, so that every participant was continually rewarded or penalized in almost exactly $25 \%$ of the trials.

In the punishment block, participants started with a virtual sum of $50 €$.In the punishment block, they lost $15 €$-cent when responding too slow or committing an error. In the reward block, participants started without any seed money and could earn a bonus of $15 €$-cent for fast responses or loose $15 €$-cent after an error. Loss and gain were indicated by a pink or green disk marked with " -0.15 " or " +0.15 ," respectively. An orange disk, marked with " -0.15 ," indicated the commission of an error. A blue disk, marked with "0.00," indicated blank feedback in trials where participants neither won nor lost. When the balance at the end of the experiment exceeded the regular participation fee of $25 €$, the extra money was paid in addition $(M=24.59 €$, gain range $=3.80-40.55 €)$.

\section{EEG recording and processing}

In general, EEG recording and preprocessing were identical to Experiment 1. The duration of feedback-locked ERP epoch was $1200 \mathrm{~ms}$, starting $200 \mathrm{~ms}$ before feedback onset. The duration of response-locked segments was $1200 \mathrm{~ms}$, starting $200 \mathrm{~ms}$ before the key press. All ERP segments were baseline-corrected with a 100ms pre-event baseline. ERPs were averaged separately for each participant, electrode, and condition.

\section{RESULTS}

\section{Performance}

To test feedback effects on conflict processing we calculated the magnitude of the Simon effect depending on preceding feedback for each experimental block. Overall error rate was $11.7 \%$ (C: $7.6 \%$, IC: $15.8 \%)$. Mean error rates in the punishment and reward blocks were 12.3 and $11.1 \%$, respectively, and did not differ significantly, $t(21)=1.1, p=0.285$. Compatibility $(2-\mathrm{C}$, IC) by feedback ( 2 - gain/loss, blank) ANOVAs on error rates were run separately for the reward and punishment block and yielded a significant main effects of compatibility in the punishment block, $F(1,21)=32.4, p<0.001$, as well as in the reward block, $F(1,21)=31.2, p<0.001$. No main effects of feedback and no interactions were significant, neither in the punishment nor in the reward block, $F$ s $<1.1$.

Only trials with correct responses preceded by correct ones and RTs $>200$ ms entered into the following RT analyses. To examine the influence of performance-contingent feedback on conflict adaptation, we applied an overall ANOVA with repeated measures on factors compatibility $(2-\mathrm{C}, \mathrm{IC})$, predecessor $(2-\mathrm{C}$, IC), reinforcement type (2 - reward block, punishment block), and feedback ( 2 - gain/loss, blank). This ANOVA revealed a main effect of compatibility, $F(1,21)=87.95, p<0.001$, a conflict adaptation effect, reflected in a significant compatibility $\times$ predecessor interaction, $F(1,21)=103.55, p<0.001$, and a four-way compatibility $\times$ predecessor $\times$ reinforcement type $\times$ feedback interaction, $F(1,21)=11.33, p<0.01$. The three-way interaction of compatibility $\times$ predecessor $\times$ reinforcement type was not significant, $F<1$.

Following up on the four-way interaction, we calculated ANOVAs for each reinforcement type (reward and punishment block) with the factors compatibility (2 - C, IC), predecessor (2 - C, IC), and feedback (2 - gain/loss, blank). In the reward block, this analysis yielded a significant main effect of compatibility, $F(1,21)=40.2, p<0.001$, and a conflict adaptation effect as expressed in the interaction of compatibility and predecessor, $F(1,21)=88.6, p<0.001$. The threeway interaction of predecessor $\times$ compatibility $\times$ feedback was significant as well, $F(1,21)=6.3, p<0.05$, indicating that conflict adaptation was more pronounced after gain feedback than after blank feedback (see Table 2), $t(21)=2.5$, $p<0.05$. 
Table 2 | Reaction times (RT) and error rates (ER) as a function of condition in Experiment 2.

\begin{tabular}{|c|c|c|}
\hline Trial type & RT in ms (SD) & ER (SD) \\
\hline \multicolumn{3}{|c|}{ REWARD BLOCK; AFTER BLANK FEEDBACK } \\
\hline Compatible preceding compatible (cC) & $302(38.4)$ & $3.4(3.3)$ \\
\hline Incompatible preceding compatible $(\mathrm{iC})$ & $345(35.6)$ & $10.9(7.3)$ \\
\hline Compatible preceding incompatible (cl) & $366(44.7)$ & $21.5(8.7)$ \\
\hline Incompatible preceding incompatible (il) & $330(48.6)$ & $8.0(4.0)$ \\
\hline Simon effect & $25(20.5)$ & $8.1(6.5)$ \\
\hline Conflict adaptation effect & 79 (35.5) & $21.0(13.0)$ \\
\hline \multicolumn{3}{|c|}{ REWARD BLOCK; AFTER GAIN FEEDBACK } \\
\hline Compatible preceding compatible (cC) & $299(36.6)$ & $3.0(4.1)$ \\
\hline Incompatible preceding compatible (iC) & $347(34.6)$ & $13.0(8.4)$ \\
\hline Compatible preceding incompatible (cl) & $372(38.5)$ & $24.6(13.6)$ \\
\hline Incompatible preceding incompatible (il) & $325(45.1)$ & $6.6(4.3)$ \\
\hline Simon effect & $25(18.3)$ & $8.0(7.6)$ \\
\hline Conflict adaptation effect & $95(54.6)$ & 27.9 (16.4) \\
\hline \multicolumn{3}{|c|}{ PUNISHMENT BLOCK; AFTER BLANK FEEDBACK } \\
\hline Compatible preceding compatible (cC) & $297(32.4)$ & $3.9(4.0)$ \\
\hline Incompatible preceding compatible $(\mathrm{iC})$ & $344(34.9)$ & $13.3(9.2)$ \\
\hline Compatible preceding incompatible (cl) & $371(37.1)$ & $24.4(10.2$ \\
\hline Incompatible preceding incompatible (il) & $326(38.9)$ & $7.0(4.7)$ \\
\hline Simon effect & $29(17.8)$ & $7.7(6.3)$ \\
\hline Conflict adaptation effect & 93 (33.9) & $26.8(14.4$ \\
\hline \multicolumn{3}{|c|}{ PUNISHMENT BLOCK; AFTER LOSS FEEDBACK } \\
\hline Compatible preceding compatible (cC) & $303(41.7)$ & $3.5(5.8)$ \\
\hline Incompatible preceding compatible (iC) & $350(38.3)$ & $13.8(9.1)$ \\
\hline Compatible preceding incompatible (cl) & $373(38.3)$ & $25.1(9.3)$ \\
\hline Incompatible preceding incompatible (il) & $334(42.8)$ & $8.8(8.9)$ \\
\hline Simon effect & $29(16.5)$ & $9.1(8.7)$ \\
\hline Conflict adaptation effect & $86(52.1)$ & $26.6(16.0$ \\
\hline
\end{tabular}

The ANOVAs for the punishment block yielded a main effect of compatibility, $F(1,21)=93.7, p<0.001$, and a significant conflict adaptation effect (compatibility $\times$ predecessor), $F(1,21)=88.6$, $p<0.001$. Furthermore, a main effect of feedback was present, $F(1,21)=7.6, p=0.012$, reflecting generally faster RTs following blank feedback than after punishment. Importantly and in contrast to the reward block no interaction of conflict adaptation with feedback was apparent, $F<1$.

As compared to blank feedback $(79 \mathrm{~ms})$ conflict adaptation after gain feedback $(95 \mathrm{~ms}$ ) was more pronounced in the reward block; however, conflict adaptation after gain feedback in the reward block did not differ significantly from that after loss feedback in the punishment block ( $86 \mathrm{~ms}), t(21)=1.3, p=0.22$. Comparing gain feedback in the reward block with loss feedback in the punishment block refers to different portions of the RT distribution, hence, the $25 \%$ fastest response in the reward block were compared to the $25 \%$ slowest responses in the punishment block. To test whether conflict adaptation is generally enlarged for fast responses in the current trial we directly compared the $25 \%$ fastest responses between reinforcement blocks and did not find a significant difference in conflict adaptation, $F<1$.

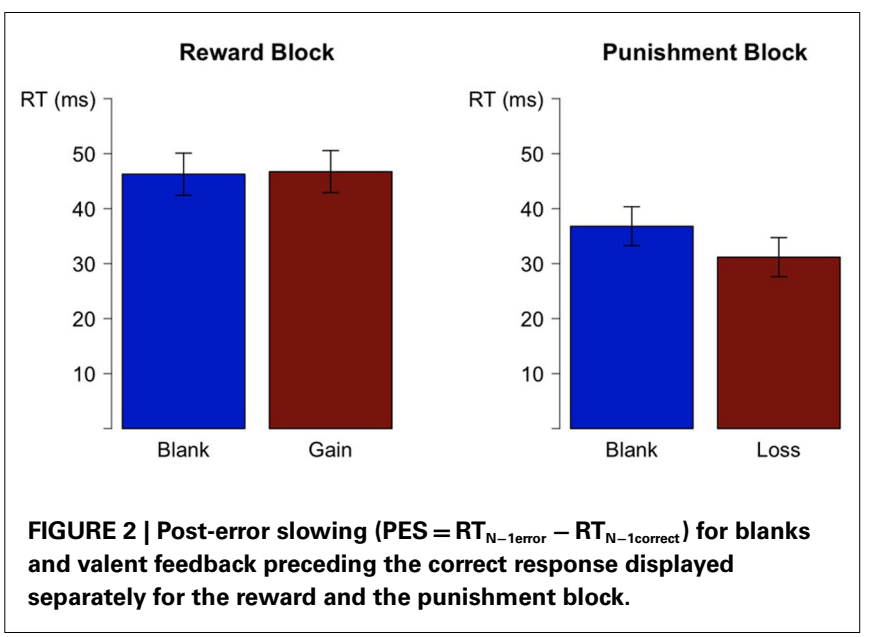

We also tested whether PES was affected by a context of punishment or reward. PES ( $\mathrm{PES}=\mathrm{RT}_{\mathrm{N}-1 \text { error }}-\mathrm{RT}_{\mathrm{N}-1 \text { correct }}$ ) was calculated by subtracting trials with correct responses preceded by a correct response from those preceded by an error. In order to avoid that the directly preceding feedback confounds with general block effects, we compared trials following correct and erroneous trials after blank feedback. PES was larger in the reward block as compared to the punishment block (see Figure 2), $t(21)=2.2, p<0.05$. The within-blocks comparisons of PES after gain or loss feedback, respectively, with PES after blank feedback (see Figure 2) were neither significant in the punishment block, $t(20)=1.5, p=0.16$, nor in the reward block, $t(21)=1.7$, $p=0.088$.

\section{Event-related potentials}

The FRN was calculated as a peak-to-peak measure at FCz electrode (see Figure 3), following the procedure of Holroyd et al. (2003). We determined the negative peak between 150 and $300 \mathrm{~ms}$ following stimulus onset and marked the preceding positive peak as the beginning of the FRN. We calculated the peak-to-peak amplitude for all four conditions and applied a repeated measures ANOVA with the factors reinforcement type $(2$ - reward block, punishment block) and feedback (2- gain/loss, blank). There was a main effect of reinforcement type, $F(1,21)=8.96, p<0.01$, and an interaction of reinforcement type and feedback, $F(1,21)=13.49$, $p=0.001$. Post hoc $t$-tests indicated larger FRN amplitudes to loss than blank feedback within the punishment block, $t(21)=2.11$, $p<0.05$, as well as smaller FRN amplitudes to gain than blank feedback within the reward block, $t(21)=4.5, p<0.001$. Further, gain feedback led to smaller amplitudes compared to loss feedback, $t(21)=3.87, p=0.001$.

The ERN was quantified in ERPs synchronized to incorrect button presses by detecting the minimum at $\mathrm{FCz}$ within a timewindow from 0 to $100 \mathrm{~ms}$. ERN amplitudes were larger in the reward block as compared to the punishment block (see Figure 4), $t(21)=2.31, p<0.05$.

\section{DISCUSSION}

Gains and losses showed differential effects on feedback-related brain potentials in Experiment 2. We can, therefore, safely 


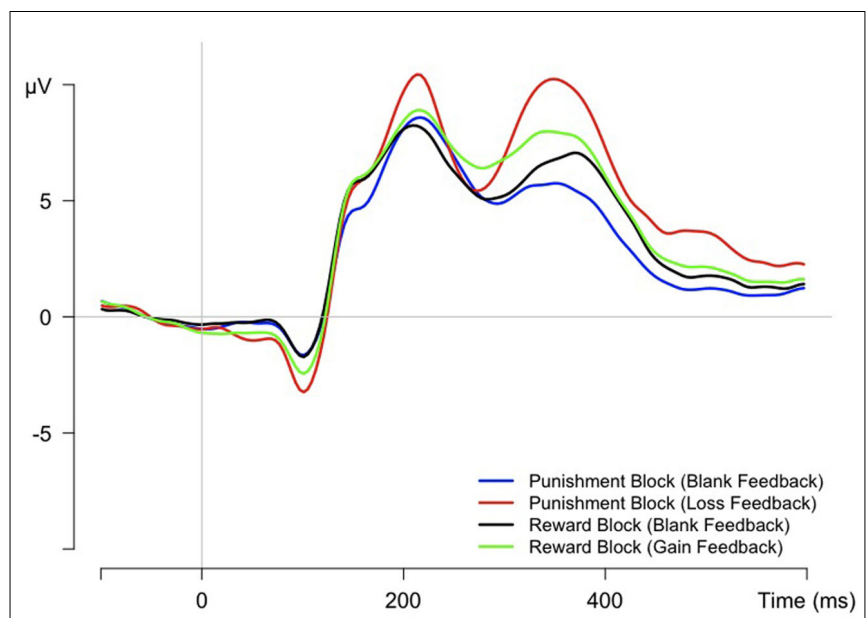

FIGURE 3 | Feedback-related grand average ERPs at FCz electrode time-locked to feedback signal presentation after correct trials.

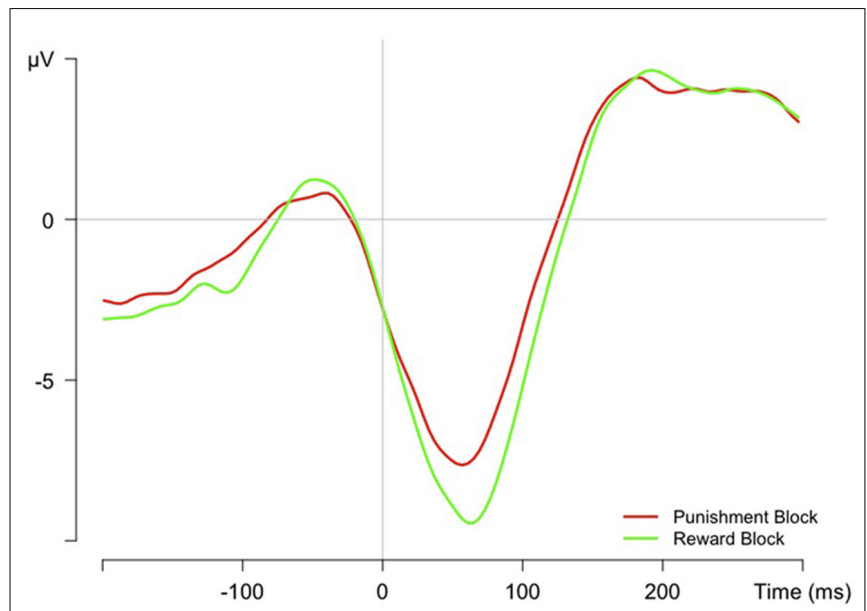

FIGURE 4 | The error-related negativity as grand average ERPs at FCz electrode time-locked to the error response.

conclude that motivationally salient stimuli were effective. In contrast to Experiment 1, we observed an affective modulation of conflict adaptation. Importantly, conflict adaptation was enhanced after gains in the reward block, whereas in the punishment block conflict adaptation was unaffected by feedback. This finding is at variance with the reports by van Steenbergen et al. $(2009,2010)$ that conflict adaptation is reduced by positive affect but is in line with the suggestion that top-down cognitive control is enhanced by DA bursts (Miller and Cohen, 2001) induced by reward. Although we observed a short-term effect of reward the overall motivational state - that is the context of reward or punishment, which varied between experimental halves - did not alter conflict adaptation.

Moreover, the ERN was enlarged and PES was enhanced in the reward as compared to the punishment block. The enlarged ERN with reward is in line with some previous studies (Larson et al.,
2006; Holroyd and Coles, 2008; Ogawa et al., 2011) but is at variance with others that reported smaller ERNs under positive affect as induced by embodied emotions (Wiswede et al., 2009b) or larger ERNs with negative affect induced by IAPs pictures (Wiswede et al., 2009a). The latter studies, however, had not shown effects of affective induction on behavior, casting doubt on whether performance monitoring was involved.

Ogawa et al. (2011) who found reduced ERN amplitudes when feedback consisted in admonishments suggested that the personal relevance of the feedback might be a modulating factor for the processing of the errors. In their study, the generator of the ERN in the admonishment condition was located in a more rostral portion within the ACC, which has been related to the affective aspects of error processing. Activation in the rostral ACC has been suggested to inhibit processes in the dorsal ACC (Bush et al., 2000). Such interpretation is supported by assumptions of current appraisal theories of emotion, as for instance the Component Process Model by Scherer $(2001,2010)$. In these frameworks, personal relevance serves as a major criterion for the elicitation and differentiation of emotions.

\section{GENERAL DISCUSSION}

The present study aimed at testing whether motivational states and short-term effects of reward and punishments affect conflict control and performance monitoring. According to the conflict monitoring account (Botvinick, 2007), conflicts are negative experiences that trigger strategic adjustments in cognitive processing in order to avoid future conflicts.

For Flanker tasks van Steenbergen et al. (2009) reported that positive affect, presumably elicited by non-contingent gain signals, reduces conflict adaptation. Against the background of the conflict monitoring account, these authors suggested that conflict adaptation was reduced because positive affect counteracts the negative experience of a conflict. In a closely related design, using the Simon interference task, we did not replicate the findings of van Steenbergen and colleagues. Conflict adaptation was not at all modulated by motivational state of the context although gain signalrelated ERPs clearly showed that win and loss were differentially registered.

In our second experiment, gain and loss were contingent upon performance. Here, they reliably influenced conflict adaptation, which was in the reward block larger after a gain relative to a blank feedback. In the punishment block conflict adaptation did not differ after a loss compared with blank feedback. Moreover, conflict adaptation after gain in the reward block and after loss in the punishment block did not differ. One could therefore as well assume that blank feedback reduced conflict adaptation in the reward block. Anyway, this finding contradicts those of van Steenbergen et al. (2009) who reported less adaptation after gain signals.

\section{CONFLICT ADAPTATION}

There might be several reasons for the discrepancy between our findings and those of van Steenbergen et al. (2009, 2010). First, effects of emotion on conflict adaptation might be as task specific as the affective consequences of cognitive conflicts, as shown by Schacht et al. (2010) who found psychophysiological emotion 
effects only for the Go/Nogo but not for the Simon task. Second, the discrepancy might be due to the fact that in our experiments direct repetitions were excluded in the conflict adaptation analysis, whereas - according to their description - this seems not have been the case in the studies of van Steenbergen et al. (2009). Not excluding conditions with especially fast responses due to direct repetitions includes a confound with conflict adaptation (Mayr et al., 2003). Third, differences in the timing of feedback signals between van Steenbergen et al. (2009) study and ours may have contributed to the discrepancies. Whereas van Steenbergen et al. (2009) presented their feedback directly after the response, we inserted a 500-ms interval. These differences in timing of feedback might have failed to interfere with conflict adaptation in the present study, although the ERP measures in our study indicated that positive feedback differed in processing from neutral or negative feedback. Inconsistent findings between the reports by van Steenbergen et al. $(2009,2010)$ and the present study do preclude strict conclusions about the role of positive affect in conflict adaptation.

\section{ERROR-RELATED ADAPTATION}

In addition to the immediate impact of rewards on conflict adaptation, the second experiment revealed an effect of general motivational state on performance monitoring in error processing. Positive affect increased the ERN and PES. The enlarged ERN under positive affect confirms previous reports (Larson et al., 2006; Holroyd and Coles, 2008) and might be due to a mismatch between a positive affective context and the error, which, in turn, might induce more conservative response strategies. An increased ERN is often associated with improvements in performance monitoring (Larson et al., 2007, 2009; Olvet and Hajcak, 2008). Better monitoring of errors might facilitate compensatory control mechanism improving performance in the subsequent trial (Gehring and Fencsik, 2001) resulting in increased PES. Although a relationship between ERN, performance monitoring, and subsequent PES seems to be plausible, this is - to our knowledge - the first study, which shows that positive affect modulates behavioral measures of error processing such as PES.

\section{CONFLICT VS. ERROR-RELATED ADAPTATION}

The results of Experiment 2 indicate increased performance monitoring in errors and conflict control under positive affect. The original conflict monitoring account (Botvinick et al., 2001) suggested one common mechanism underlying both conflict and error monitoring. Thus, both should result in identical or at least similar strategic adjustments. Recently, this suggestion is being called into question by reports in support of independent adaptive mechanisms. For instance, Notebaert and Verguts (2010) investigated conflict and error adaptation in a task-switching paradigm using two interference tasks. They showed that PES generalized across tasks whereas conflict adaptation did not, arguing against a unitary adaptation mechanism.

Hikosaka and Isoda (2010) suggested that adjacent medialfrontal brain areas are involved in two complementary modes of cognitive control. One operates on performance failures consisting in errors or unexpectedly high rewards ("reward prediction error"). Both situations seem to alter midbrain DA release and, hence, modulate activation of ACC neurons. This mode was called retroactive because the control processes were triggered by behavioral performance. The other control mode was called proactive because here an external cue indicated a new task context for response selection. It was suggested that the proactive control mode is mediated by the pre-supplementary motor area (pre-SMA). In contrast to the ACC, pre-SMA processing is not directly related to the midbrain DA system; only indirect relations exist via basal ganglia output targeting the pre-SMA.

Ullsperger and King (2010) extended this approach by assuming that the proactive control mode selectively prepares appropriate task sets and triggers conflict adaptation. Reactive control, however, enhances responsiveness to any potentially relevant stimulus. Reactive control is recruited by performance errors and triggers adaptation after errors (e.g., PES). Following up, midbrain $\mathrm{DA}$ is directly related to error adaptation via ACC processing but it is not to conflict adaptation via the pre-SMA.

Our finding that error processing is affected by motivational states could be accounted for by the proposed midbrain DA and dorsal ACC circuitries involved in reactive control. At the same time, modulations of conflict adaptation by motivational states were inconsistent between studies. Evidence for affective modulation of conflict adaptation is, therefore, only sparse. These less conclusive reports of affective modulations in conflict adaptation could be accounted for by the pre-SMA involvement in proactive control, which is not (directly) linked to the midbrain DA system probably crucial for cognitive-affective interactions.

Even when errors and conflicts show similar consequences on subsequent behavior by generally slowing responses in the upcoming event (Verguts et al., 2011), some aftereffects do dissociate. Conflicts result in more focused processing in the next trial thereby reducing potential conflicts. Errors, however, did not reduce upcoming conflicts.

\section{CONCLUSION}

Recent findings clearly speak for different adaptation mechanisms triggered by errors and conflicts. An increasing number of studies support cognitive-affective interactions in error processing. Affective modulations of conflict control are, however, less clear. We observed a temporary modulation of conflict adaptation effects only in blocks in which gain was achieved contingent upon task-performance. In contrast to van Steenbergen et al. (2009), we did not observe any effects of motivational state on conflict adaptation when gains and losses were applied non-contingent to task-performance as a lottery. Importantly, the overall context of reward or punishment did not alter conflict adaptation.

\section{ACKNOWLEDGMENTS}

This research was supported by a grant of the German Research Foundation (STU 248/3-1). 


\section{REFERENCES}

Botvinick, M. M. (2007). Conflict monitoring and decision making: reconciling two perspectives on anterior cingulate function. Cogn. Affect. Behav. Neurosci. 7, 356-366.

Botvinick, M. M., Braver, T. S., Barch, D. M., Carter, C. S., and Cohen, J. D. (2001). Conflict monitoring and cognitive control. Psychol. Rev. 108, 624-652.

Bush, G., Luu, P., and Posner, M. I. (2000). Cognitive and emotional influences in anterior cingulate cortex. Trends Cogn. Sci. (Regul. Ed.) 4, 215-222.

Egner, T., Delano, M., and Hirsch, J. (2007). Separate conflict-specific cognitive control mechanisms in the human brain. Neuroimage 35, 940-948.

Falkenstein, M., Hoormann, J., Christ, S., and Hohnsbein, J. (2000). ERP components on reaction errors and their functional significance: a tutorial. Biol. Psychol. 51, 87-107.

Fielding, J., Georgiou-Karistianis, N., Bradshaw, J., Millist, L., and White, O. (2005). No sequence dependent modulation of the Simon effect in Parkinson's disease. Brain Res. Cogn. Brain Res. 25, 251-260.

Gehring, W. J., and Fencsik, D. E. (2001). Functions of the medial frontal cortex in the processing of conflict and errors. J. Neurosci. 21, 9430-9437.

Gratton, G., Coles, M. G., and Donchin, E. (1992). Optimizing the use of information: strategic control of activation of responses. J. Exp. Psychol. Gen. 121, 480-506.

Hikosaka, O., and Isoda, M. (2010). Switching from automatic to controlled behavior: cortico-basal ganglia mechanisms. Trends Cogn. Sci. (Regul. Ed.) 14, 154-161.

Holroyd, C. B., and Coles, M. G. (2002). The neural basis of human error processing: reinforcement learning, dopamine, and the errorrelated negativity. Psychol. Rev. 109, 679-709.

Holroyd, C. B., and Coles, M. G. (2008). Dorsal anterior cingulate cortex integrates reinforcement history to guide voluntary behavior. Cortex 44, 548-559.

Holroyd, C. B., Nieuwenhuis, S., Yeung, N., and Cohen, J. D. (2003). Errors in reward prediction are reflected in the event-related brain potential. Neuroreport 14, 2481-2484.

Holroyd, C. B., Pakzad-Vaezi, K. L., and Krigolson, O. E. (2008). The feedback correct-related positivity: sensitivity of the event-related brain potential to unexpected positive feedback. Psychophysiology 45, 688-697.

Kuhl, J., and Kazen, M. (1999). Volitional facilitation of difficult intentions: joint activation of intention memory and positive affect removes Stroop interference. J. Exp. Psychol. Gen. 128, 382-399.

Larson, M. J., and Clayson, P. E. (2011). The relationship between cognitive performance and electrophysiological indices of performance monitoring. Cogn. Affect. Behav. Neurosci. 11, 159-171.

Larson, M. J., Kaufman, D. A., Kellison, I. L., Schmalfuss, I. M., and Perlstein, W. M. (2009). Double jeopardy! The additive consequences of negative affect on performancemonitoring decrements following traumatic brain injury. Neuropsychology 23, 433-444.

Larson, M. J., Kaufman, D. A., Schmalfuss, I. M., and Perlstein, W. M. (2007). Performance monitoring, error processing, and evaluative control following severe TBI. J. Int. Neuropsychol. Soc. 13, 961-971.

Larson, M. J., Perlstein, W. M., StiggeKaufman, D., Kelly, K. G., and Dotson, V. M. (2006). Affective contextinduced modulation of the errorrelated negativity. Neuroreport 17, 329-333.

Leuthold, H., and Sommer, W. (1999). ERP correlates of error processing in spatial S-R compatibility tasks. Clin. Neurophysiol. 110, 342-357.

Lu, C. H., and Proctor, R. W. (1995). The influence of irrelevant location information on performance a review of the Simon and spatial Stroop effects. Psychol. Bull. Rev. 2, 174-207.

Masaki, H., Falkenstein, M., Stürmer, B., Pinkpank, T., and Sommer, W. (2007). Does the error negativity reflect response conflict strength? Evidence from a Simon task. Psychophysiology 44, 579-585.

Mayr, U., Awh, E., and Laurey, P. (2003). Conflict adaptation effects in the absence of executive control. Nat. Neurosci. 6, 450-452.

Miller, E. K., and Cohen, J. D. (2001). An integrative theory of prefrontal cortex function. Annu. Rev. Neurosci. 24, 167-202.

Miltner, W. H. R., Braun, C. H., and Coles, M. G. H. (1997). Event-related brain potentials following incorrect feedback in a timeestimation task: evidence for a "generic" neural system for error detection. J. Cogn. Neurosci. 9, 788-798.
Notebaert, W., Houtman, F., Opstal, F. V., Gevers, W., Fias, W., and Verguts, T. (2009). Post-error slowing: an orienting account. Cognition 111, 275-279.

Notebaert, W., and Verguts, T. (2010). Conflict and error adaptation in the Simon task. Acta Psychol. (Amst) 136, 212-216.

Ogawa, K., Masaki, H., Yamazaki, K., and Sommer, W. (2011). The influence of emotions due to verbal admonishment and encouragement on performance monitoring. Neuroreport 22, 313-318.

Oldfield, R. C. (1971). The assessment and analysis of handedness: the Edinburgh inventory. Neuropsychologia 9, 97-113.

Olvet, D. M., and Hajcak, G. (2008) The error-related negativity (ERN) and psychopathology: toward an endophenotype. Clin. Psychol. Rev. 28, 1343-1354.

Praamstra, P., and Plat, F. M. (2001) Failed suppression of direct visuomotor activation in Parkinson's disease. J. Cogn. Neurosci. 13, 31-43.

Ridderinkhof, K. R., Ullsperger, M. Crone, E. A., and Nieuwenhuis, S. (2004). The role of the medial frontal cortex in cognitive control. Science 306, 443-447.

Schacht, A., Dimigen, O., and Sommer, W. (2010). Emotions in cognitive conflicts are not aversive but are task specific. Cogn. Affect. Behav. Neurosci. 10, 349-356.

Schacht, A., Nigbur, R., and Sommer, W. (2009). Emotions in go/nogo conflicts. Psychol. Res. 73 843-856.

Scherer, K. R. (2001). "Appraisal considered as a process of multi-level sequential checking," in Appraisal Processes in Emotion: Theory, Methods, Research, eds K. R. Scherer, A. Schorr, and T. Johnstone (New York: Oxford University Press), 92-120.

Scherer, K. R. (2010). "The component process model: a blueprin for a comprehensive computational model of emotion," in Blueprint for Affective Computing: A Sourcebook, eds K. R. Scherer, T. Bänziger, and E. B. Roesch (Oxford: Oxford University Press), 47-70.

Schultz, W. (1998). Predictive reward signal of dopamine neurons. J. Neurophysiol. 80, 1-27.

Simons, R. F. (2010). The way of our errors: theme and variations. Psychophysiology 47, 1-14.

Stürmer, B., Leuthold, H., Soetens, E., Schröter, H., and Sommer, W. (2002). Control over location-based response activation in the simon task: behavioral and electrophysiological evidence. J. Exp. Psychol. Hum. Percept. Perform. 28, 1345-1363.

Ullsperger, M., and King, J. (2010). Proactive and reactive recruitment of cognitive control: comment on Hikosaka and Isoda. Trends Cogn. Sci. (Regul. Ed.) 14, 191-192.

van Steenbergen, H., Band, G. P. H., and Hommel, B. (2009). Reward counteracts conflict adaptation. Psychol. Sci. 20, 1473-1477.

van Steenbergen, H., Band, G. P. H., and Hommel, B. (2010). In the mood for adaptation: how affect regulates conflict-driven control. Psychol. Sci. 21, 1629-1634.

Verguts, T., Notebaert, W., Kunde, W., and Wuhr, P. (2011). Post-conflict slowing: cognitive adaptation after conflict processing. Psychon. Bull. Rev. 18, 76-82.

Wiswede, D., Münte, T. F., Goschke, T., and Rüsseler, J. (2009a). Modulation of the error-related negativity by induction of short-term negative affect. Neuropsychologia 47, 83-90.

Wiswede, D., Münte, T. F., Kramer, U. M., and Rüsseler, J. (2009b). Embodied emotion modulates neural signature of performance monitoring. PLoS ONE 4, e5754. doi:10.1371/journal.pone.0005754

Yeung, N., Cohen, J. D., and Botvinick, M. M. (2004). The neural basis of error detection: conflict monitoring and the error-related negativity. Psychol. Rev. 111, 931-959.

Conflict of Interest Statement: The authors declare that the research was conducted in the absence of any commercial or financial relationships that could be construed as a potential conflict of interest.

Received: 29 July 2011; accepted: 31 October 2011; published online: 16 November 2011.

Citation: Stürmer B, Nigbur R, Schacht $A$ and Sommer W (2011) Reward and punishment effects on error processing and conflict control. Front. Psychology 2:335. doi: 10.3389/fpsyg.2011.00335

This article was submitted to Frontiers in Cognition, a specialty of Frontiers in Psychology.

Copyright (c) 2011 Stürmer, Nigbur, Schacht and Sommer. This is an openaccess article subject to a non-exclusive license between the authors and Frontiers Media SA, which permits use, distribution and reproduction in other forums, provided the original authors and source are credited and other Frontiers conditions are complied with. 